Data and text mining

\title{
Differentiating isoform functions with collaborative matrix factorization
}

\author{
Keyao Wang ${ }^{1}$, Jun Wang ${ }^{1}$, Carlotta Domeniconi ${ }^{2}$, Xiangliang Zhang $^{3 *}$ and \\ Guoxian $\mathrm{Yu}^{1,3 *}$
}

\author{
${ }^{1}$ College of Computer and Information Science, Southwest University, Chongqing 400715, China. \\ ${ }^{2}$ Department of Computer Science, George Mason University, Fairfax 22030, VA, USA. \\ ${ }^{3}$ Computer, Electrical, and Mathematical Sciences and Engineering Division, King Abdullah University of Science and Technology, SA \\ *xiangliang.zhang@kaust.edu.sa(X. Zhang); gxyu@swu.edu.cn(G. Yu) \\ Associate Editor: $X X X X X X X$ \\ Received on XXXXX; revised on XXXXX; accepted on XXXXX
}

\begin{abstract}
Motivation: Isoforms are alternatively spliced mRNAs of genes. They can be translated into different functional proteoforms, and thus greatly increase the functional diversity of protein variants (or proteoforms). Differentiating the functions of isoforms (or proteoforms) helps understanding the underlying pathology of various complex diseases at a deeper granularity. Since existing functional genomic databases uniformly record the annotations at the gene-level, and rarely record the annotations at the isoform-level, differentiating isoform functions is more challenging than the traditional gene-level function prediction. Results: Several approaches have been proposed to differentiate the functions of isoforms. They generally follow the multi-instance learning paradigm by viewing each gene as a bag and the spliced isoforms as its instances, and push functions of bags onto instances. These approaches implicitly assume the collected annotations of genes are complete and only integrate multiple RNA-seq datasets. As such, they have compromised performance. We propose a data integrative solution (called DisoFun) to Differentiate isoform Functions with collaborative matrix factorization. DisoFun assumes the functional annotations of genes are aggregated from those of key isoforms. It collaboratively factorizes the isoform data matrix and gene-term data matrix (storing Gene Ontology (GO) annotations of genes) into low-rank matrices to simultaneously explore the latent key isoforms, and achieve function prediction by aggregating predictions to their originating genes. In addition, it leverages the PPI network and GO structure to further coordinate the matrix factorization. Extensive experimental results show that DisoFun improves the AUROC (area under the receiver-operating characteristic curve) and AUPRC (area under the precision-recall curve) of existing solutions by at least $7.7 \%$ and $28.9 \%$, respectively. We further investigate DisoFun on four exemplar genes (LMNA, ADAM15, BCL2L1, and CFLAR) with known functions at the isoform-level, and observed that DisoFun can differentiate functions of their isoforms with $90.5 \%$ accuracy.
\end{abstract}

Availability: The code of DisoFun is available at mlda.swu.edu.cn/codes.php?name=DisoFun

Contact: xiangliang.zhang@kaust.edu.sa(X. Zhang); gxyu@swu.edu.cn(G. Yu)

Supplementary information: Supplementary data are available at Bioinformatics online.

\section{Introduction}

More than $90 \%$ of human multi-exon genes undergo alternative splicing, which significantly increases the transcriptome and proteome complexity (Pan et al., 2008; Wang et al., 2008). Alternative splicing produces different isoforms from a single gene through different mechanisms, such as exon skipping, mutual exclusion of exons, alternative 5' donor site, alternative 3 ' acceptor site, and intron retention. The isoforms are finally translated into proteoforms (Smith et al., 2013), which perform various biological functions of a living cell. However, existing functional genome databases (i.e., Gene Ontology (GO, 2016) and KEGG (Kanehisa et al., 2016)) 
typically follow the convention of collectively storing all the functions of gene products as associated to the originating gene. In other words, these databases store functional knowledge at the gene-level. But the biological functions of a multi-exon gene are not identically inherited by it spliced isoform(s), which may even have opposite functions (Smith $e t$ al. 2013). For example, two isoforms, $\mathrm{Bcl}-\mathrm{x}(\mathrm{S})$ and $\mathrm{Bcl}-\mathrm{x}(\mathrm{L})$, of the $\mathrm{B}$-cell lymphoma-x (BCL2L1) gene have opposite biological functions, namely proapoptotic and anti-apoptotic (Revil et al., 2007). Increasing studies show that aberrant alternative splicing causes many cellular abnormalities and leads to various cancers (i.e., colorectal cancer and spinal muscular atrophy), and autism spectrum disorders (Skotheim et al., 2007; Xiong et al., 2015). Therefore, differentiating the intrinsic functions of isoforms can contribute to a deeper understanding of both the molecular basis of diverse genetic diseases and the evolution of phenotypic complexity ( $\mathrm{Li}$ et al., 2014a). However, compared to the widely-studied gene/protein function prediction, how to differentiate the functions of isoforms is still a rarely studied and more challenging topic.

Isoform function prediction faces two key challenges: (1) The lack of large-scale genomic data at the isoform-level, and (2) The unavailability of a systematic catalog of isoform-level functional annotations. With the development of RNA-seq technology, large-scale high-resolution transcript-level expression data can be easily collected (Wang et al., 2009) These RNA-seq datasets, and the accompanied quantitative tools (Teng et al., 2015), enable the differentiation of isoforms' functions. However, isoforms still lack systematic 'ground-truth' functional annotations.

Recently, several multiple instance learning (MIL) (Dietterich et al., 1997) based solutions have been proposed to tackle the challenge of bare annotations of isoforms (Eksi et al., 2013; Li et al., 2014b; Luo et al., 2017; Shaw et al., 2019; Yu et al., 2019; Chen et al., 2019). The basic principle of MIL is that if a bag is annotated with a label, then at least one of its instances is annotated with the same label; on the other hand, if a bag does not have a certain label, none of its instances has that label as well (Dietterich et al., 1997; Zhou et al., 2012). This principle coincides with the convention of GO annotations of genes, which collectively associates all the functions of gene products with the originating genes (GO, 2016). Based on this principle, existing solution generally view each gene as a bag, and isoforms of the gene as it instances, and then push the gene-level annotations to the isoform-level. Eksi et al. (2013) adopted a multiple-instance support vector machine (miSVM) (Andrews et al., 2003) to differentiate the functions of isoforms of Mouse. miSVM utilizes gene-level annotations, isoform expression data, and gene-isoform relations to generate classification models at the isoform-level. Panwar et al. (2016) further used miSVM to annotate the functions of isoforms of Human. Li et al. (2014b) developed a nove multi-instance label propagation method (iMILP) to predict functions of isoforms. iMILP first constructs multiple functional association network of isoforms from multiple RNA-seq datasets, and uniformly initializes the annotations of isoforms based on the GO annotations of their originating genes. After that, iMILP iteratively updates the annotations of isoforms in these networks using label propagation, and allows all qualified isoforms to inherit a positive annotation of a gene under a 'democratic' learning principle until convergence. Luo et al. (2017) proposed a sparse simplex projection based approach (WLRM) to efficiently solve the problem of highly nontrivial non-convex and non-smooth optimization in miSVM, and applied it for isoform function prediction. Shaw et al. (2019) proposed a deep learning based method (DeepIsoFun) to predict isoform functions. DeepIsoFun uses auto-encoders to extract features from expression data and domain adaptation to provide additional labeled training data, and then transfer the knowledge of gene functions to the prediction of isoform functions. These methods implicitly assume that the functional annotations of genes are complete, and only integrate multiple RNA-seq datasets, while ignoring the quite relevant and important protein-protein interaction data and Gene Ontology. Chen et al. (2019) introduced another deep learning based solution (DIFFUSE), which leverages deep neural network on the genomic sequences to predict the functions of isoforms and refines the predictions by conditional random field on the co-expression data in a coordinated fashion. Yu et al. (2019) recently introduced an approach called IsoFun, which shows an improved accuracy by fusing proteinprotein interaction data and Gene Ontology. Isofun applies random walks on a heterogeneous network composed with genes, isoforms and GO terms to predict GO annotations of isoforms. However, alike iMILP, IsoFun initializes all the annotations of a gene to its isoforms. In addition, to ensure each annotation inherited by isoforms, it clamps those annotations to the most 'responsible' isoform of that gene during the random walk process, which overrides other responsible isoforms. Given the functional diversity of isoforms, these wrongly initialized and clamped annotations compromise the performance of IsoFun.

In this paper, we introduce a collaborative matrix factorization based data fusion solution, called DisoFun, to differentiate isoform functions. The main idea of DisoFun and its workflow are illustrated in Figure 1. DisoFun assumes the functional annotations of genes are aggregated from key isoforms, and the known functions of genes are dispersed to key isoforms and consequent to other isoforms. To meet this assumption, we jointly factorize the isoform expression data matrix $\mathbf{X}$ (derived from multiple RNA-seq datasets) and the gene-term association matrix (Y) (derived from the GO annotations of genes) into low-rank matrices ( $\mathbf{K}$ $\mathbf{U}$ and $\mathbf{V}$ ). The first two low-rank matrices of the first factorization $\mathbf{X}=\mathbf{U K}^{T}$ aim at identifying $k$ latent 'key isoforms' $(\mathbf{K})$ and their relations with other isoforms $(\mathbf{U})$. The third low-rank matrix $\mathbf{V}$ (which stores the latent functions of these key isoforms) and the shared matrix $\mathbf{U}$ in the second factorization $\mathbf{Y}=\mathbf{A} \mathbf{U} \mathbf{V}^{T}$ aim at first expanding the latent functions of key isoforms to all isoforms via $\mathbf{U} \mathbf{V}^{T}$, and then at aggregating these latent annotations to their originated genes via the geneisoform relation matrix $\mathbf{A}$. By enforcing the aggregated annotations being consistent with the known annotations of genes, we can reversely push back the annotations to key isoforms and coordinate the identification of key isoforms. Given the importance of protein interaction data and Gene Ontology in gene function prediction, and the incomplete GO annotation of genes, DisoFun further leverages PPI networks and GO hierarchy to replenish the annotations of genes and those of key isoforms. As a result, the functional annotations of key isoforms and those of other isoforms are collaboratively predicted by fusing data from gene-level, isoformlevel, GO hierarchy, and inter-relations between them. Finally, DisoFun approximates the target isoform-term association matrix as $\mathbf{F}=\mathbf{U V}^{T}$ We observe that DisoFun can also be used to combine amino acids (nucleotide) data and other biological data.

We conducted experiments on 298 Human RNA-seq datasets collected from ENCODE (ENCODE Project Consortium, 2012), and found that DisoFun achieves significantly better results than other related and competitive methods (Eksi et al., 2013; Li et al., 2014b; Luo et al., 2017; Shaw et al., 2019; Yu et al., 2019). Both the PPI network and the GO hierarchy improve the performance of isoform function prediction. In addition, DisoFun can be effective on a wide range of key isoforms. We also applied DisoFun for the prediction of isoforms' functions of LMNA, ADAM15, BCL2L1, and CFLAR, which are known. Our findings show that DisoFun can accurately (accuracy $=90.5 \%=19 / 21$ ) differentiate the functions of these isoforms.

\section{Materials and Method}

\subsection{Materials}

Suppose there are $n$ genes; the $i$-th gene produces $m_{i} \geq 1$ isoforms, and the total number of isoforms is $m=\sum_{i=1}^{n} m_{i} . \mathbf{A} \in \mathbb{R}^{n \times m}$ is the 


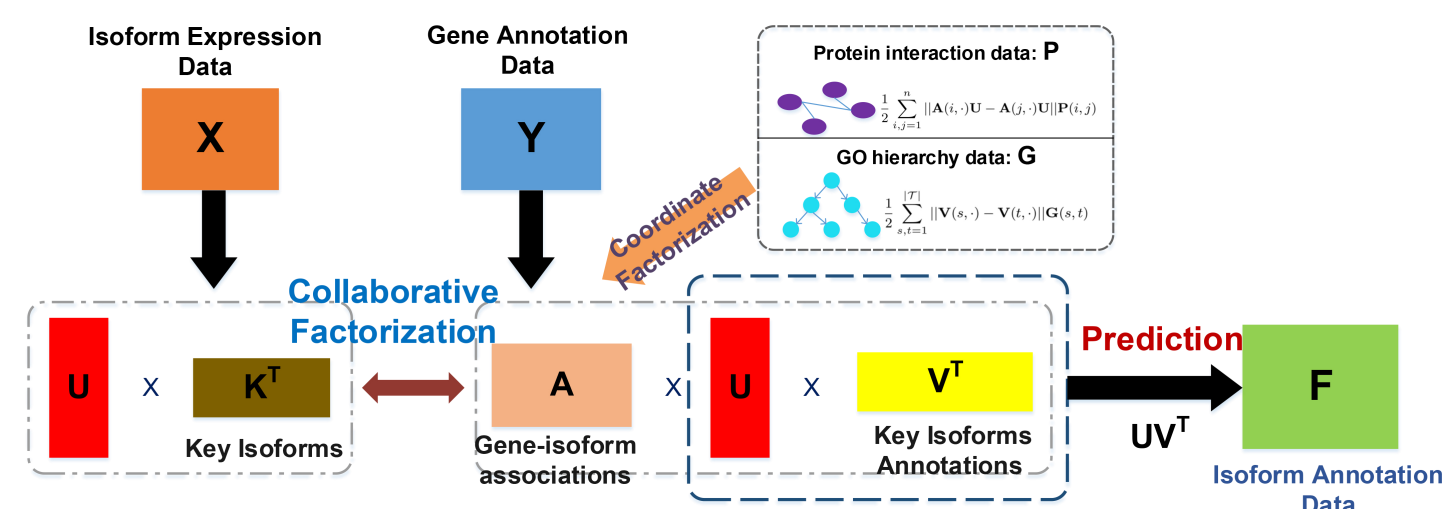

Fig. 1. Workflow of DisoFun. The isoform expression data matrix $\mathbf{X}$ and the gene function annotation data matrix $\mathbf{Y}$ are collaboratively factorized into low-rank matrices ( $\mathbf{U}, \mathbf{K}$ and $\mathbf{V})$ $\mathbf{K}$ denotes the latent key isoforms, $\mathbf{V}$ embeds the latent functions of the key isoforms, and $\mathbf{U}$ encodes the relationships between other isoforms and key isoforms. $\mathbf{U}$ is shared between $\mathbf{X}$ and $\mathbf{Y}$ for collaboration. A encodes the associations between isoforms and their originating genes; it can disperse the functions of genes to isoforms and aggregate the functions of isoforms. The protein interaction data and the GO hierarchy are combined to further coordinate the collaboration, and to differentiate isoform functions.

relational data matrix between $n$ genes and $m$ isoforms; $\mathbf{A}(i, j)=1$ if the $j$-th isoform originated from the $i$-th gene, $\mathbf{A}(i, j)=0$ otherwise.

We adopt the widely-used Fragments Per Kilobase of exon per Million fragments mapped fragments (FPKM) values to quantify the expression of isoforms. Let $\mathbf{X} \in \mathbb{R}^{m \times d}$ store the FPKM values of $m$ isoforms with respect to $d$ samples. To construct $\mathbf{X}$, we downloaded 596 RNA-seq runs (of total 298 samples from different tissues and conditions) of Human from the ENCODE project (access date: 2018-11-10). These datasets are heterogeneous in terms of library preparation procedures and sequencing platform. As done by Li et al. (2014b), we follow the below procedure to control the quality of the original data.

(1) HISAT2 (v.2-2.1.0) (Kim et al., 2015) is first used to align the shortreads of each RNA-seq dataset of the Human genome (build GRCh38.90) from Ensemble.

(2) A GTF annotation file of the same build is used with an option of no-novel-junction. Then, we use StringTie (v.1.3.3b) (Pertea et al., 2015) to calculate the relative abundance of the transcript as FPKM values. We separately compute the FPKM values of a total of 57,964 genes with 219,288 isoforms for each sample.

(3) The FPKM values of very short isoforms are exceptionally higher Therefore, the isoforms with less than 100 nucleotides are discarded. For example, the tRNA usually has less than 100 nucleotides.

(4) To further control the quality of isoforms, we use known proteincoding gene names to map those genes obtained in step (3). Finally, we obtained $n=11,868$ genes with $m=25,939$ isoforms, whose values are stored in the corresponding data matrix $\mathbf{X} \in \mathbb{R}^{m \times d}$. We normalize $\mathbf{X}$ by column, $\mathbf{X}(:, j)_{n o r}=\mathbf{X}(:, j) . / \max (\mathbf{X}(:, j))$. For convenience, we use $\mathbf{X}$ to represent $\mathbf{X}(:, j)_{\text {nor }}$ for subsequent formula.

We collected the PPI data from BioGrid ${ }^{1}$, which is a publicly accessible database of physical and genetic interactions of molecules with an exhaustive curation. If the $i$-th protein (gene) interacts with the $j$-th protein, we set $\mathbf{P}(i, j)$ as the strength value of the interaction; otherwise, $\mathbf{P}(i, j)=0$. We want to remark that $\mathbf{P} \in \mathbb{R}^{n \times n}$ is not a symmetric matrix, since the interaction between two proteins can be directed.

We downloaded the ontology file from the GO website ${ }^{2}$ to initialize the GO structural data $\mathbf{G} \in \mathbb{R}^{|\mathcal{T}| \times|\mathcal{T}|}(\mathcal{T}$ includes all the studied GO terms). The GO terms are structured via a direct acyclic graph; the child term is a further division of its parent term, and the child term often describes a more specific biological function. Each term can be viewed as a functional label. Based on the hierarchical relationship between terms, we initialize

${ }^{1}$ https://thebiogrid.org/download.php

${ }^{2}$ http://geneontology.org/page/download-ontology
G as follows:

$$
\mathbf{G}(s, t)=\left\{\begin{array}{l}
1, \text { if } t \in \operatorname{child}(s) \\
0, \text { otherwise }
\end{array}\right.
$$

where $\operatorname{child}(s)$ includes all the direct child terms of $s$.

We also downloaded the contemporary GO annotation file of Human from the same website as the ontology file, and used $\mathbf{Y} \in \mathbb{R}^{n \times|\mathcal{T}|}$ to store the currently known annotations of $n$ genes with respect to $|\mathcal{T}|$ GO terms. $\mathbf{Y}$ is initialized as follows:

$$
\mathbf{Y}(i, t)=\left\{\begin{array}{l}
1, \text { if } t \in \mathcal{T}_{i} \\
0, \text { otherwise }
\end{array}\right.
$$

where $\mathcal{T}_{i} \subseteq \mathcal{T}$ includes all the terms annotated to the $i$-th gene

Existing methods (Eksi et al., 2013; Li et al., 2014b; Luo et al., 2017) mainly use $\mathbf{X}, \mathbf{Y}$, and $\mathbf{A}$ under the MIL framework to predict isoform functions. They simply assume that the collected annotations of genes are complete. But in fact, the $\mathrm{GO}$ annotations of proteins are often incomplete (Huntley et al., 2014). Furthermore, they disregard the important and curated PPI data and the hierarchical relationship between GO terms, both of which contribute to replenish the annotations of genes and to push back these annotations to individual isoforms. DeepIsoFun (Shaw et al., 2019) additionally uses the GO hierarchy to train the model, but it's still limited to expression data. For these reasons, we introduce an integrative approach to leverage PPI data, GO hierarchy, and multiple RNA-seq datasets to differentiate isoform function.

\subsection{Collaborative matrix factorization}

GO follows the convention of collectively assigning the functions of gene products to the originating gene. If a GO term is annotated to a gene, this term should be assigned to at least one isoform spliced from that gene. In other words, each functional annotation of genes is responsible (or triggered) by an isoform. We term these responsible isoforms as key isoforms, which inherit the functions of genes. As a result, the annotations of other isoforms can be obtained based on their relations with those key isoforms. Therefore, the function prediction task is transformed into the question of how we can leverage multi-level biological data, and how we can explore the key isoforms. To handle this task, we introduce a collaborative matrix factorization objective:

$$
\mathcal{O}^{\prime}(\mathbf{U}, \mathbf{K}, \mathbf{V})=\left\|\mathbf{X}-\mathbf{U K}^{T}\right\|_{F}^{2}+\left\|\mathbf{Y}-\mathbf{A} \mathbf{U} \mathbf{V}^{T}\right\|_{F}^{2}
$$

where $\|\cdot\|_{F}^{2}$ is the Frobenius norm. $\mathbf{K} \in \mathbb{R}^{d \times k}, \mathbf{U} \in \mathbb{R}^{m \times k}$, and $\mathbf{V} \in \mathbb{R}^{|\mathcal{T}| \times k}$ are three low-rank matrices; they separately encode the 
feature information of $k$ latent key isoforms, the intra-relation between key isoforms and all the isoforms, and latent functions of key isoforms. The first factorization in Eq. (3) is the classical nonnegative matrix factorization (Lee et al., 1999). It is equivalent to clustering when viewing $\mathbf{K}$ as the encoding of $k$ cluster centroids, and $\mathbf{U}$ as the encoding of the relations between isoforms and the centroids (Ding et al., 2008). From this perspective, we can consider the centroids as the latent key isoforms. The second factorization has two interleaved objectives: (i) expanding the latent functions of key isoforms to other isoforms via $\mathbf{U} \mathbf{V}^{T}$, and then aggregating them to the originating genes via $\mathbf{A} \mathbf{U V}^{T}$; (ii) dispersing the known annotations of genes to isoforms via factorizing $\mathbf{Y}$. The interaction is achieved by sharing $\mathbf{U}$ among $\mathbf{X}$ and $\mathbf{Y}$. The function transfer between genes and isoforms is bridged by $\mathbf{A}$.

Current GO annotations of genes are still rather incomplete. They are biased by the research interests of biologists and experiment ethics on animals (Huntley et al., 2014). But also Eq. (3), like other cousin solutions (Eksi et al., 2013; Li et al., 2014b; Luo et al., 2017), does not take into consideration the incomplete annotations of genes and the domain knowledge of the GO hierarchy. In addition, the readily available and highly relevant PPI data is disregarded.

Interacting proteins are more likely to share functions (Schwikowski et al., 2000), and they may have similar relationships with respect to $k$ key isoforms. As such, we define a regularization term to employ the protein interaction data and to guide the factorization of $\mathbf{U}$ as follows:

$$
\begin{aligned}
\operatorname{Reg}_{1}(\mathbf{A U}) & =\frac{1}{2} \sum_{i, j=1}^{n}\|\mathbf{A}(i, \cdot) \mathbf{U}-\mathbf{A}(j, \cdot) \mathbf{U}\|_{F}^{2} \mathbf{P}(i, j) \\
& =\operatorname{tr}\left(\mathbf{U}^{T} \mathbf{A}^{T}\left(\mathbf{D}_{p}-\mathbf{P}\right) \mathbf{A} \mathbf{U}\right)=\operatorname{tr}\left(\mathbf{U}^{T} \mathbf{A}^{T} \mathbf{L}_{p} \mathbf{A} \mathbf{U}\right)
\end{aligned}
$$

where $\mathbf{A}(i, \cdot) \mathbf{U} \in \mathbb{R}^{1 \times k}$ encodes the inter-relation between the $i$-th gene and $k$ key isoforms, $\mathbf{D}_{p} \in \mathbb{R}^{n \times n}$ is a diagonal matrix with $\mathbf{D}_{p}(i, i)=\sum_{j=1}^{n} \mathbf{P}(i, j), \mathbf{L}_{p}=\mathbf{D}_{p}-\mathbf{P}$ is the graph Laplacian matrix of $\mathbf{P}$, and $\operatorname{tr}(\cdot)$ denotes the matrix trace operator. Minimizing the above regularization can enforce proteins with interactions related to similar key isoforms having similar functions. This minimization also guides the replenishment of missing annotations of proteins via multiplying $\mathbf{A}(i, \cdot) \mathbf{U}$ with $\mathbf{V}$. As a result, the issue of incomplete annotations is alleviated and the replenished annotations can be finally pushed back to isoforms.

The GO hierarchy carries important biological knowledge and contributes to reliable and consistent function predictions of gene product (GO, 2016; Huntley et al., 2014; Fu et al., 2016; Shaw et al., 2019). Given this, we use the GO hierarchy to guide the prediction of latent functions of key isoforms and introduce another regularization as follows:

$$
\begin{aligned}
\operatorname{Reg}_{2}(\mathbf{V}) & =\frac{1}{2} \sum_{s, t=1}^{|\mathcal{T}|}\|\mathbf{V}(s, \cdot)-\mathbf{V}(t, \cdot)\|_{F}^{2} \mathbf{G}(s, t) \\
& =\operatorname{tr}\left(\mathbf{V}^{T}\left(\mathbf{D}_{g}-\mathbf{G}\right) \mathbf{V}\right)=\operatorname{tr}\left(\mathbf{V}^{T} \mathbf{L}_{g} \mathbf{V}\right)
\end{aligned}
$$

where $\mathbf{D}_{g} \in \mathbb{R}^{|\mathcal{T}| \times|\mathcal{T}|}$ is a diagonal matrix with $\mathbf{D}_{g}(s, s)=$ $\sum_{t=1}^{|\mathcal{T}|} \mathbf{G}(s, t)$, and $\mathbf{L}_{g}=\mathbf{D}_{g}-\mathbf{G}$. If $t$ and $s$ are two closely related GO terms, then they should annotate to the same set of key isoforms. Therefore, minimizing the above regularization term can guide the assignment of closely related GO terms to similar key isoforms.

Based on the above, we formalize the objective functions of DisoFun as follows:

$$
\begin{aligned}
\mathcal{O}(\mathbf{U}, \mathbf{K}, \mathbf{V}) & =\left\|\mathbf{X}-\mathbf{U K}^{T}\right\|^{2}+\left\|\mathbf{Y}-\mathbf{A} \mathbf{U V}^{T}\right\|^{2} \\
& +\lambda \operatorname{tr}\left(\mathbf{V}^{T} \mathbf{L}_{g} \mathbf{V}\right)+\beta \operatorname{tr}\left(\mathbf{U}^{T} \mathbf{A}^{T} \mathbf{L}_{p} \mathbf{A} \mathbf{U}\right)
\end{aligned}
$$

where $\lambda \geq 0$ and $\beta \geq 0$ are the scalar parameters to balance the importance of the two regularization terms. We can observe that the target low-rank matrices ( $\mathbf{U}, \mathbf{K}$, and $\mathbf{V}$ ) are collaboratively learned among $\mathbf{X}, \mathbf{Y}, \mathbf{P}$, and $\mathbf{G}$, which are seamlessly merged to differentiate the GO annotations of isoforms.

Based on the optimization technique of standard NMF (Lee et al., 1999), we adopt an iterative optimization procedure to alternatively optimize one of $\mathbf{U}, \mathbf{K}$, and $\mathbf{V}$ while fixing the other two as constants, until convergence. Due to space limitation, the alternative optimization procedure and convergency proof are provided in the Supplementary file. Our empirical study shows that DisoFun converges in 40 iterations.

\subsection{Isoform/gene function prediction}

Suppose $\mathbf{K}^{*}, \mathbf{V}^{*}$, and $\mathbf{U}^{*}$ are optimized low-rank matrices. Based on the intra-relation between $m$ isoforms and these latent $k$ key isoforms, we can accomplish the isoform function prediction as follows:

$$
\mathbf{F}=\mathbf{U}^{*} \mathbf{V}^{* T}
$$

Similarly, based on the gene-isoform inter-relations, we can predict the functions of genes via $\mathbf{F}=\mathbf{A} \mathbf{U}^{*} \mathbf{V}^{* T}$.

\section{Results}

\subsection{Experimental setup}

To quantify the performance of DisoFun in differentiating isoform functions, we downloaded two releases of GO annotation files of Human archived in different years from the GO website ${ }^{3}$. The historical GOA file was archived on 2016-05-01, and the recent GOA file was archived on 2018-11-24. We adopt the historical to recent experimental protocol, which is adopted by CAFA (Jiang et al., 2016) and is more challenging and practical than the widely adopted cross-validation protocol. Particularly, we train DisoFun on the historical (released in 2016) GOA file, and validate its predictions on the recent (released in 2018) GOA file with the new annotations archived between 2016 and 2018. To avoid the impact of GO structure changes, we also downloaded the contemporary GO files ${ }^{4}$ and used the shared GO structure for the experiments. The ontology file includes the hierarchical relationship between GO terms, and is organized in branches: Biological Process Ontology (BPO), Molecular Function Ontology (MFO) and Cellular Function Ontology (CCO). The annotation file records associations between $\mathrm{GO}$ terms and genes, which indicate the relevant genes that carry the biological functions described by the related GO terms. We use the GO hierarchy to augment the annotations of genes. In particular, if a term is annotated to a gene, then its ancestor terms are inherently annotated to the same gene. The augmented annotations are then used to initialize the gene-term association matrix $\mathbf{Y}$. To avoid circular prediction, annotations with evidence code 'IEA' (inferred from electronic annotations) were excluded. miSVM makes predictions separately for each GO term and is very time-consuming for a large set of GO terms. For comparison, we filter out sparse GO terms that are associated to very few $(<10)$ genes. The processed GO annotations of genes and isoforms are listed in Table 1. From this table, we can see that many new annotations of genes were appended during the two-year interval.

Due to the lack of ground-truth annotations for isoforms, we have to aggregate the isoform-level predictions to the gene-level, and then refer to gene ground-truth annotations for comparison. This surrogate evaluation was also adopted by previous studies (Eksi et al., 2013; Luo et al., 2017; Yu et al., 2019). In addition, for comprehensive evaluation, we also tested DisoFun on single-isoform genes (as done by iMILP (Li et al., 2014b)) and on four exemplar genes, whose isoform-level annotations are known.

\footnotetext{
${ }_{3}$ http://geneontology.org/ page/download-annotations/

${ }^{4}$ http://geneontology.org/page/download-ontology/
} 
Table 1. Statistics of GO annotations, genes, and isoforms used for experiments on Human.

\begin{tabular}{l|ccc}
\hline$\sharp$ genes $(n)$ & \multicolumn{3}{|c}{11,868} \\
$\sharp$ isoforms $(m)$ & \multicolumn{3}{|c}{25,939} \\
Dimensions of isoforms $(d)$ & \multicolumn{3}{|c}{298} \\
\hline & BPO & MFO & CCO \\
\hline history $(2016-05-01)$ & 580,521 & 101,216 & 221,248 \\
recent $(2018-11-24)$ & 660,468 & 119,363 & 256,167 \\
$\sharp$ terms $(|\mathcal{T}|)$ & 4,141 & 811 & 601 \\
\hline
\end{tabular}

Evaluation metrics: Many evaluation metrics have been suggested to measure the performance of gene function prediction. For a comprehensive comparison, we adopt four metrics used by CAFA2 (Jiang et al., 2016), namely AUROC, AUPRC, Fmax, and Smin. AUROC first calculates the area under receiver operating curve of each term, and then takes the average value of these areas as a whole to quantify the performance. $A U P R C$ calculates the average value of the area under the precisionrecall curve of each term, and then quantify the performance with the average value. Fmax is the overall maximum harmonic mean of recall and precision across all possible thresholds on F. Smin uses information theoretic analogs of precision and recall by referring to the GO hierarchy to compute the minimum semantic distance between the predictions and ground-truth across all possible thresholds on $\mathbf{F}$. The formal definition of these metrics can be found in (Jiang et al., 2016). The smaller the values of Smin is, the better the performance is; the opposite trend holds for the other three metrics. We want to remark that these metrics evaluate the performance of function prediction from different perspectives, one method cannot always outperform another one across all these metrics.

Comparing methods: We compare our method against miSVM, MISVM (Eksi et al., 2013), WLRM (Luo et al., 2017), iMILP (Li et al., 2014b), DeepIsoFun (Shaw et al., 2019) and IsoFun (Yu et al., 2019). These comparing methods were reviewed in the Introduction. miSVM identifies the top 25\% isoforms as 'responsible' isoforms of a gene with respect to a GO term. On the other hand, MISVM considers only the isoform with the maximum score as the 'responsible' isoform.

In the following experiments, unless otherwise specified, we fix $k=$ $200, \lambda=100, \beta=1$, and the number of iterations to optimize lowrank matrices as 50 . The input parameters of these comparing methods are directly specified as the authors reported or optimized in the suggested ranges.

\subsection{Component contribution analysis}

To investigate the contribution components of DisoFun, we introduce three variants of DisoFun: DisoFun(nGP), DisoFun(nG), and DisoFun(nP). DisoFun(nGP) neither uses GO hierarchy, nor the PPI data; DisoFun(nG) and DisoFun(nP) exclude the GO hierarchy and PPI data, respectively. The differences between these three variants and DisoFun are summarized in Table 2. Figure 2 reports the Fmax and Smin values of DisoFun and its variants.

Table 2. Differences between DisoFun and the variants of DisoFun.

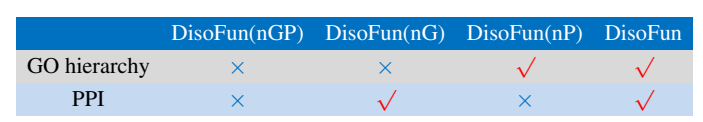

DisoFun has a largely superior performance compared to its variants. This observation supports our motivation to integrate the available PPI data and GO hierarchy data. DisoFun(nG) sometimes obtains better results than DisoFun(nGP), especially on BPO. This is because the PPI replenishes the missing annotation of proteins. DisoFun(nG) often loses to DisoFun(nP), which suggests the GO hierarchy contributes much more than the PPI data in differentiating isoform functions. That is because the more specific functional annotations are gradually annotated to genes, which often correspond to descendants of the terms (annotations) already annotated to these genes (Yu et al., 2015).
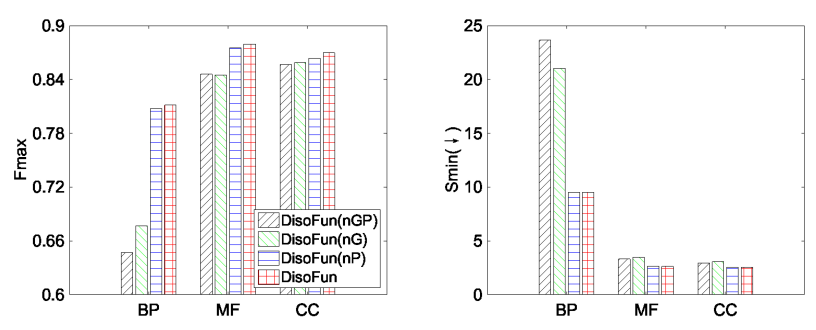

Fig. 2. Results of DisoFun and its variants.

DisoFun has three important parameters $(\lambda, \beta$, and $k$, see Eq. (6) in Section 3.2), which may affect its performance. We conducted additional experiments to investigate the sensitivity of DisoFun to $\lambda, \beta$, and $k$. The obtained results show that when $\lambda \in[100,1000], \beta \in[0.01,1]$ and $k>$ 100, DisoFun can achieve the best performance. Due to page limitation, the experimental results and analysis are provided in Figure S1, Figure S2 and Section 1 of the Supplementary file.

\subsection{Prediction results at the gene-level}

Since mi-SVM, MI-SVM and DeepIsoFun cannot adopt the historical to recent experimental protocol, we only use the recent data (released in 2018) for experiment. We randomly divided the genes (and their isoforms) into a training set (80\%) and a validation set (20\%). Due to the prohibitive runtime of mi-SVM and MI-SVM on such a large number of GO terms, we exclude the terms annotated to fewer than 30 genes, and the too general terms annotated to more than 300 genes. After that, the numbers of GO terms used for the experiments are 2004 (BPO), 376 (MFO) and 318 (CCO), respectively. The functional annotations of genes in the validation set are considered as unknown during training and prediction. To be fair, we ensure that each GO term is annotated to at least one gene in the training set. To speed up the training of miSVM and MISVM, as done by WLRM, we select negative genes twice as positive ones for each term to train the model. Unlike the history to recent experimental protocol adopted in Section 3.2, for experiments in this Section, when a gene does not have any annotation, its functions can only be predicted by other genes with high similarity. Therefore, we reduce the weight of GO hierarchy by fixing $\lambda=1$ and report the experimental results in Table 3.

DisoFun is significantly better than the comparing methods across most evaluation metrics. More specifically, DisoFun improves the AUROC, AUPRC and Fmax of the second best method (IsoFun) by at least $7.7 \%$, $28.9 \%$ and $73.4 \%$, respectively. Fmax is the gene-centric metrics, AUROC and $A U P R C$ are GO term-centric metrics. This prominent improvement shows that DisoFun can more reliably predict the functions of genes (isoforms) from both the gene and GO term perspectives. Two factors contribute to this improvement: (i) DisoFun can leverage multi-level data to more comprehensively capture the view of isoforms and their function mechanisms; (ii) Collaborative matrix factorization helps to coherently explore and predict the latent functions of key isoforms, the functions of other isoforms, and thus the functions of genes. MiSVM, MISVM and WLRM also try to explore the responsible isoforms, but they all lose to DisoFun, since they only merge expression data, and assume the collected GO annotations are complete. They neither make concrete use of the GO hierarchy, nor of the important protein interaction data. DeepIsoFun combines the expression data and the GO hierarchy. As a result, it generally 
Table 3. Experimental results of five-fold cross-validation at the gene-level. $\downarrow$ means the lower the value, the better the performance is. $\bullet / \circ$ indicates DisoFun performing significantly better/worse than the other comparing method, where significance is measured using a pairwise $t$-test at $95 \%$ level.

\begin{tabular}{|c|c|c|c|c|c|c|c|c|}
\hline & & $\mathrm{miS}$ & M & $\mathrm{WI}$ & $P$ & Fun & Isc & \\
\hline \multirow{3}{*}{ AUROC } & & $029 \pm$ & $075 \pm$ & $209 \pm$ & $645 \pm$ & $038 \bullet$ & $075 \bullet$ & 098 \\
\hline & & & & $4 \bullet$ & & $3 \bullet$ & & \\
\hline & $\mathrm{CO}$ & $39 \bullet$ & $278 \pm$ & $0.6409 \pm$ & $0.6155 \pm$ & 0.6109 & $0.6944=$ & 0.769 \\
\hline \multirow{3}{*}{ AUPRC } & & $06 \bullet$ & $095 \pm 0$ & $095 \pm 0$ & $0.0100 \pm 0$ & 0.0136 & $0.0164 \pm$ & 0.021 \\
\hline & FO & $092 \pm 0.0020 \bullet$ & $086 \pm 0$ & $0.0104 \pm$ & $0.0154 \pm$ & $0.0155=$ & $0.0161 \pm$ & $0.0266 \pm 0.0025$ \\
\hline & $\mathrm{CO}$ & $0176 \pm$ & $158 \pm$ & 0.0194 & $0.0153 \pm$ & 0.023 & $0.0264 \pm$ & 0.053 \\
\hline \multirow{3}{*}{ Fmax } & $\mathrm{O}$ & $.0412 \pm$ & $0559 \pm$ & 0.0242 & $0.0769=$ & 0.048 & $0.0898 \pm 0$ & $0.1580 \pm 0.0003$ \\
\hline & MFO & $0.0448 \pm$ & $0.0420 \pm c$ & $0.0297 \pm($ & $0.0735 \pm 0$ & $0.0018 \bullet$ & $0.0854 \pm 0.0043 \bullet$ & $0.1480 \pm 0.0093$ \\
\hline & $\mathrm{CCO}$ & $0.0610 \pm 0.0200 \bullet$ & $0.0711 \pm 0.0268 \bullet$ & $0.0461 \pm 0.0002 \bullet$ & $0.1232 \pm 0.0014 \bullet$ & $0.0735 \pm 0.0008 \bullet$ & $0.1305 \pm 0.0032 \bullet$ & $0.2467 \pm 0.0083$ \\
\hline \multirow{3}{*}{$\operatorname{Smin} \downarrow$} & ) & - & - & $3 \bullet$ & $6 \bullet$ & $78 \circ$ & 7297 & 10 \\
\hline & MFO & $79 \bullet$ & $0 \bullet$ & 95• & $7 \bullet$ & $178 \circ$ & $293 \bullet$ & $=0.0255$ \\
\hline & $\mathrm{CO}$ & $2 \bullet$ & 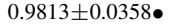 & $\bullet$ & & 10 & $88 \bullet$ & \\
\hline
\end{tabular}

obtains better results than other comparing methods, but still loses to DisoFun, since it disregards the PPI data. DeepIsoFun uses GO hierarchy to build the model and Smin is defined with respect to GO hierarchy, as such DeepIsoFun obtains a better Smin than DisoFun. Since we do not use domain data to train DeepIsoFun, DeepIsoFun gives a lower AUROC than miSVM. WLRM usually has a better performance than miSVM and MISVM. That is because WLRM assigns different weights to the isoform of each gene, whereas miSVM and MISVM treat the isoforms equally. Due to this equal treatment of isoforms, IsoFun loses to DisoFun, although they both leverage the PPI and GO data, and account for the missing annotations of genes. Another cause for this loss is that IsoFun is misled by wrongly clamped annotations during the random walk process. Al these comparing methods do not show a high AUROC and AUPRC. This is because the isoform expression data are still too sparse and the isoformlevel annotations are rare; as such, they both prohibit the effectiveness of these methods. We observe that DisoFun manifests lower results than those in Section 3.2. That is because we train DisoFun on the historical (released in 2016) GOA file, and validate its predictions on the recent (released in 2018) GOA file with new annotations archived between 2016 and 2018 (called history to recent). For this evaluation, we pushed back the history annotations, so the prediction results of DisoFun are better To be consistent with other comparing methods, in this section, we use the recent data for five-fold cross validation, without pushing annotations back.

We further divide the GO terms into three subgroups ([30, 100], [101, 200], [201, 300]). Particularly, [30, 100] means each term in that subgroup is annotated to at least 30 genes but at most 100 genes. Next, we compute the average AUROC and AUPRC for GO terms in each subgroup and report the results in Figure S3 of the Supplementary file. We observe that DisoFun can achieve the highest AUPRC and AUROC across different granularity of GO terms. The AUPRC in subgroup [201, 300] is better than those in other two subgroups, since AUPRC is sensitive to class-imbalance and GO terms in this subgroup are more balanced and have more member genes. On the other hand, AUROC is less sensitive to class-imbalance, so its values in different subgroups are relatively stable.

We also recorded the runtime costs of all the methods and convergence trend of DisoFun. Although DisoFun does not show the smallest runtime cost, it always achieves the most prominent predictions at both the genelevel and isoform-level. As for the convergency trend, DisoFun generally converges in 40 iterations across different GO branches. These results and analysis are provided in Table S1, Figure S4 and Section 3 of the Supplementary file.

\subsection{Prediction results at the isoform-leve}

Although isoforms lack ground-truth annotations, an isoform can inherit the functions of a single-isoform gene, who produces one isoform only.
There are 2782 single-isoform genes in our collected data. We use the annotations of the single-isoform genes to approximately evaluate the predictions of miSVM, iMILP, IsoFun and DisoFun at the isoform-level under the history to recent setting, and report the resulting $A U R O C$ and $A U P R C$ in Fig. 3. Other comparing methods can not make the isoform level (instance-label) prediction under this setting, so they are excluded for experiments.

DisoFun again has a better performance than IsoFun, iMILP and miSVM, which shows its advantage in differentiating the functions of isoforms. iMILP only propagates annotations of isoforms on the isoformlevel functional association network, and it does not account for the GO hierarchy and PPI data. For similar reasons, miSVM loses to DisoFun, although it also explores the key isoforms for function prediction. Since IsoFun initializes the annotations of a gene to all its isoform, and may wrongly clamp annotations to an isoform, it loses to DisoFun. The performance margin achieved by DisoFun against IsoFun, miSVM and iMILP not only proves the effectiveness of integrating multi-level data for isoform function prediction, but also suggests the advantage of collaboratively factorizing the data to explore latent key isoforms and their functions.

To further study the capability of DisoFun, IsoFun and miSVM in differentiating the functions of isoforms originated from the same gene, we take four exemplar multi-isoform genes, namely LMNA (Lamin A/C), ADAM15 (ADAM Metallopeptidase Domain 15), CFLAR (CASP8 and FADD Like Apoptosis Regulator), and BCL2L1 (Bcl-2-like 1), whose isoform-level functions were studied in wet-lab experiments. Table 4 and Table S2-S3 of the Supplementary file reveal the predictions of DisoFun, IsoFun and miSVM on the isoforms of these genes, respectively.

Table 4. The annotated (DisoFun, IsoFun or miSVM predicted) GO annotations of isoforms of LMNA. $\sqrt{ }$ indicates that the GO term is annotated (predicted) to the isoform, and $\times$ means the opposite. When the predicted association probability between a GO term and an isoform is in the top 20\% of the total isoforms of the gene, the isoform is annotated with this term. Wrong predictions are highlighted in red font.

\begin{tabular}{c|cccc}
\hline Isoform & & GO:0006612 & GO:0034446 & GO:2001257 \\
\hline \multirow{2}{*}{ lamin C } & Annotation & $\times$ & $\times$ & $\sqrt{ }$ \\
& DisoFun/IsoFun/miSVM & $\times / \times / \times$ & $\times / \times / \times$ & $\sqrt{ } / \sqrt{ } / \sqrt{ }$ \\
\hline \multirow{2}{*}{ lamin A } & Annotation & $\sqrt{ }$ & $\times$ & $\times$ \\
& DisoFun/IsoFun/miSVM & $\sqrt{ } / \sqrt{ } / \sqrt{ }$ & $\sqrt{ } / \times / \sqrt{ }$ & $\times / \times / \times$ \\
\hline \multirow{2}{*}{ progerin } & Annotation & $\times$ & $\sqrt{ }$ & $\times$ \\
& DDisoFun/IsoFun/miSVM & $\times / \times / \times$ & $\sqrt{ } / \times / \times$ & $\times / \sqrt{ } / \sqrt{ }$ \\
\hline
\end{tabular}

LMNA is a component of the nuclear lamina, which has an important status in nuclear assembly, chromatin organization, nuclear membrane, and telomere dynamics. Lopez-Mejia et al. (2014) reported 

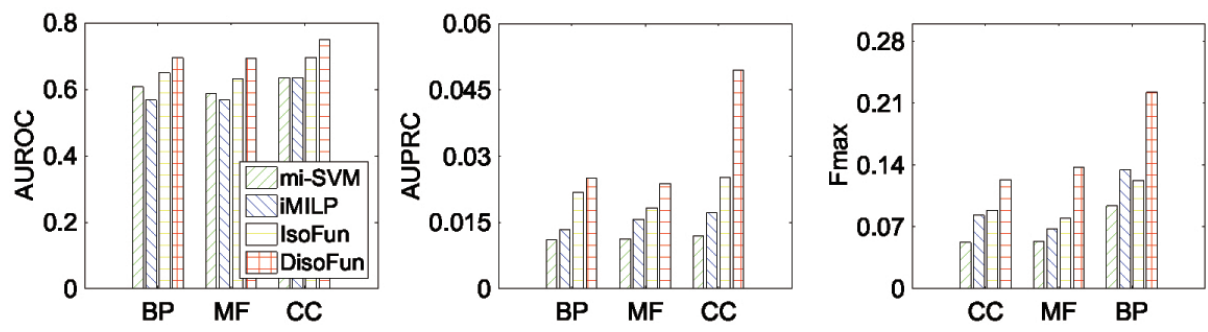

Fig. 3. Results of miSVM, iMILP, IsoFun and DisoFun at the isoform-level.

the experimental functional validation of three main isoforms of LMNA, lamin A, progerin, and lamin C. Lamin $\mathrm{C}$ expression is mutually exclusive with the splicing of lamin A and progerin isoforms, and occurs by alternative polyadenylation. LMNA encodes functionally distinct isoforms that have opposing effects on energy metabolism and lifespan in mammals. Particularly, lamin C expressing mice live longer, show decreased energy metabolism, increased weight gain, and reduced respiration. In contrast, progerin-expressing mice show increased energy metabolism and are lipodystrophic. Based on this, we used three GO terms (GO:0006612: Protein targeting to membrane, GO:0034446: Substrate adhesion-dependent cell spreading, and GO:2001257: Regulation of cation channel activity) related to the three isoforms to test the differential ability of DisoFun. In Table 4, DisoFun can differentiate the functions of isoforms almost perfectly, and the overall accuracy on the three isoforms is $88.9 \%(8 / 9)$. As for IsoFun and miSVM, their accuracy are $77.8 \%(7 / 9)$ and $66.7 \%(6 / 9)$, respectively. The accuracy of DisoFun on other three genes are $100 \%, 75 \%, 100 \%$, and those of IsoFun are $75 \%, 50 \%, 75 \%$, and those of miSVM are $75 \%, 100 \%, 25 \%$.

Based on these prediction results, we can confirm that different isoforms spliced from the same gene indeed have distinct functions. In summary, DisoFun can more accurately $(90.5 \%=19 / 21)$ differentiate the functions of isoforms than IsoFun $(71.4 \%=15 / 21)$ and miSVM $(66.7 \%=14 / 21)$. These case studies again support the advantage of DisoFun to IsoFun and miSVM.

We further compare DisoFun with the more recent DIFFUSE (Chen et al., 2019). Since DIFFUSE does not provide the corresponding expression data but the co-expression network of isoforms, as such DisoFun cannot use the network data shared by DIFFUSE for isoform function prediction. In addition, DisoFun does not use sequence data of isoforms and domain data, and the used training data for DisoFun cannot be directly used by DIFFUSE. So we do not report the results of DIFFUSE in Section 3.3. For comparison, we test DisoFun on 14 isoforms of 6 genes, which were used to validate DIFFUSE. We report the results in Table 5 and Table 6. DIFFUSE also gives the probability prediction, and it relies on the prediction scores of different isoforms the same gene, but it does not clarify how they transform the probability scores into binary prediction. Since different genes have different number of isoforms, it is inappropriate to set an absolute value as the cut off. Given that, we again fixed the cut off to $20 \%$ for DisoFun, whose association scores are above the average score of total isoforms of the same gene, and directly used the originally reported results of DIFFUSE for comparison. We can see that DisoFun and DIFFUSE have the same number of wrong predictions. DisoFun has three false negative, while DIFFUSE has three false positive. DIFFUSE mainly uses binary predictors and initializes the annotations of a gene to all isoforms spliced from this gene, as such DIFFUSE inclines to give false positive predictions. On the other hand, DisoFun makes use of a single predictor for all GO terms without such initialization, given such a large number of candidate terms and the sparse annotations of genes, it gives false negative predictions. How to further reduce the false negative/positive prediction is an interesting future pursue.
Table 5. The literature supported (DisoFun or DIFFUSE predicted) GO annotations of isoforms of ACE, ACMSD and GCH1 with respect to GO:0046872. $\sqrt{ }$ indicates that the GO term is annotated (predicted) to the isoform, and $\times$ means the opposite. Wrong predictions are highlighted in red font.

\begin{tabular}{c|cccc}
\hline GO:0046872 & & GO:0046872 & DisoFun & DIFFUSE \\
\hline \multirow{2}{*}{ ACE } & P12821-1 & $\sqrt{ }$ & $\sqrt{ }$ & $\sqrt{ }$ \\
& P12821-3 & $\sqrt{ }$ & $\sqrt{ }$ & $\sqrt{ }$ \\
\hline \multirow{2}{*}{ ACMSD } & Q8TDX5-1 & $\sqrt{ }$ & $\times$ & $\sqrt{ }$ \\
& Q8TDX5-2 & $\times$ & $\times$ & $\sqrt{ }$ \\
\hline \multirow{3}{*}{ GCH1 } & P30793-1 & $\sqrt{ }$ & $\sqrt{ }$ & $\sqrt{ }$ \\
& P30793-2 & $\times$ & $\times$ & $\times$ \\
& P30793-4 & $\times$ & $\times$ & $\times$ \\
\hline
\end{tabular}

Table 6. The literature supported (DisoFun or DIFFUSE predicted) GO annotations of isoforms of ACE, ACMSD and GCH1 with respect to GO:0005634. $\sqrt{ }$ indicates that the GO term is annotated (predicted) to the isoform, and $\times$ means the opposite. Wrong predictions are highlighted in red font.

\begin{tabular}{c|cccc}
\hline GO:0005634 & & GO:0005634 & DisoFun & DIFFUSE \\
\hline \multirow{2}{*}{ ADK } & P55263-1 & $\sqrt{ }$ & $\times$ & $\sqrt{ }$ \\
& P55263-2 & $\times$ & $\times$ & $\sqrt{ }$ \\
\hline \multirow{3}{*}{ ACMSD } & O95831-1 & $\sqrt{ }$ & $\sqrt{ }$ & $\sqrt{ }$ \\
& O95831-3 & $\times$ & $\times$ & $\sqrt{ }$ \\
& O95831-4 & $\times$ & $\times$ & $\times$ \\
\hline \multirow{2}{*}{ GCH1 } & Q12972-1 & $\sqrt{ }$ & $\times$ & $\sqrt{ }$ \\
& Q12972-3 & $\times$ & $\times$ & $\times$ \\
\hline
\end{tabular}

\section{Conclusions}

Differentiating the functions of alternatively spliced isoforms can pave the way for explaining the proteome complexity and various complex diseases at a higher resolution than at the gene-level. Due to the lack of ground-truth annotations of isoforms, existing gene function prediction methods cannot be applied to the isoform-level. This is a rarely studied and hard challenge. To tackle this problem we develop a data integrative model called DisoFun. DisoFun follows the principle that the functional annotations of genes are aggregated from 'responsible' (or key) isoforms. DisoFun aims at dispersing the known functions of genes to key isoforms and consequently to other isoforms. To reach this target, DisoFun collaboratively factorizes multi-level data matrices to explore the functions of latent key isoforms, to replenish the annotations of genes and further disperse them to isoforms in a cooperative manner. Experimental results show that DisoFun outperforms other related and representative solutions. The study also confirms that the protein interaction data and the GO hierarchy should be merged to differentiate isoform functions accurately.

There are several avenues to further improve the performance of DisoFun, for example, combining multiple gene-level, transcript-level and phenotype heterogeneous data sources. 


\section{Funding}

This work is supported by NSFC (61872300 and 61873214), Fundamental Research Funds for the Central Universities (XDJK2019B024), Natural Science Foundation of CQ CSTC (cstc2018jcyjAX0228).

\section{References}

Andrews, S. et al (2003). Support vector machines for multiple-instance learning. Advances in Neural Information Processing Systems, pp.577584.

Chen, H. et al (2019). DIFFUSE: predicting isoform functions from sequences and expression profiles via deep learning. Bioinformatics, 35(14), i284-i294.

Dietterich, T.G. et al (1997). Solving the multiple instance problem with axis-parallel rectangles. Artificial Intelligence, 89(1-2), 31-71.

Ding, C. et al (2008). Convex and semi-nonnegative matrix factorizations. IEEE Trans. on Pattern Analysis and Machine Intelligence, 32(1), pp 45-55.

Eksi, R. et al (2013). Systematically Differentiating Functions for Alternatively Spliced Isoforms through Integrating RNA-seq Data. PLoS Computational Biology, 9(11), e1003314.

ENCODE Project Consortium. (2012). An integrated encyclopedia of DNA elements in the human genome. Nature, 489(7414), 57.

Fu, G. et al (2016). NegGOA: negative GO annotations selection using ontology structure. Bioinformatics, 32(19), 2996-3004.

Gene Ontology Consortium. (2016). Expansion of the Gene Ontology knowledgebase and resources. Nucleic Acids Research, 45(D1), D331 D338.

Huntley, R.P. et al (2014). Understanding how and why the Gene Ontology and its annotations evolve: the GO within UniProt. GigaScience, 3: 4.

Jiang, Y. et al (2016). An expanded evaluation of protein function prediction methods shows an improvement in accuracy. Genome Biology, 17: 184

Kanehisa, M. et al (2016). KEGG: new perspectives on genomes, pathways, diseases and drugs. Nucleic Acids Research, 45(D1), D353D361.

Kim, D. et al (2015). HISAT: a fast spliced aligner with low memory requirements. Nature Methods, 12(4), 357-360.

Lee, D.D. et al (1999). Learning the parts of objects by non-negative matrix factorization. Nature, 401(6755), 788 .

Li, H. et al (2014a). The emerging era of genomic data integration for analyzing splice isoform function. Trends in Genetics, 30(8), 340-347.

Li, W. et al (2014b). High-resolution functional annotation of human transcriptome: predictingisoform functions by a novel multiple instancebased label propagationmethod. Nucleic Acids Research, 42(6), e39.
Lopez-Mejia, I.C. et al (2014). Antagonistic functions of LMNA isoforms in energy expenditure and lifespan. EMBO Reports, 15(5), 529-539.

Luo, T. et al (2017). Functional annotation of human protein coding isoforms via non-convex multi-instance learning. Proceedings of the 23rd ACM SIGKDD International Conference on Knowledge Discovery and Data Mining, pp.345-354.

Pan, Q. et al (2008). Deep surveying of alternative splicing complexity in the human transcriptome by high-throughput sequencing. Nature Genetics, 40(12), 1413.

Panwar, B. et al (2016). Genome-wide functional annotation of human protein-coding splice variants using multiple instance learning. Journal of Proteome Research, 15(6), 1747-1753.

Pertea, M. et al (2015). StringTie enables improved reconstruction of a transcriptome from RNA-seq reads. Nature Biotechnology, 33(3), 290295.

Revil, T. et al (2007). Protein Kinase C-Dependent Control of Bcl-x Alternative Splicing. Molecular and Cellular Biology, 27(24), 84318441.

Schwikowski B. et al (2000). A network of proteinšCprotein interactions in yeast. Nature Biotechnology, 18(12), 1257.

Shaw, D. et al (2019). DeepIsoFun: A deep domain adaptation approach to predict isoform functions. Bioinformatics, 35(15), 2535-2544.

Skotheim, R.I. et al (2007). Alternative splicing in cancer: Noise, functional, or systematic? International Journal of Biochemistry \& Cell Biology, 39(7-8), 1432-1449.

Smith, M. et al (2013). Proteoform: a single term describing protein complexity. Nature Methods, 10(3), 186.

Teng, M. et al (2015). A benchmark for RNA-seq quantification pipelines. Genome Biology, 2016, 17: 74.

Wang, E.T. et al (2008). Alternative isoform regulation in human tissue transcriptomes. Nature, 456(7221), 470-476.

Wang Z. et al (2009). RNA-Seq: a revolutionary tool for transcriptomics. Nature Reviews Genetics, 10(1), 57.

Xiong, H.Y. et al (2015). The human splicing code reveals new insights into the genetic determinants of disease. Science, 347(6218), 1254806.

Yu, G. et al (2015). Predicting protein function via downward random walks on a gene ontology. BMC Bioinformatics, 16: 271.

Yu, G. et al (2019). Isoform function prediction based on bi-random walks on a heterogeneous network. Bioinformatics, 99(1), 1-7.

Zhou, Z.H. et al (2012). Multi-instance multi-label learning. Artificial Intelligence, 176(1), 2291-2320. 